\title{
28 Research Square \\ Identification Of Immune Networks Influenced By \\ Mir-200c In IDH1-Mutant Lower-Grade Gliomas \\ Using WGCNA
}

\section{Yunxia Duan}

Hubei University of Medicine

Linan Li

Xiangyang Central Hospital

Pengzhu Li

Ludwig Maximilians University Munich

Peng Duan

Hubei University of Medicine

Hua Liu

Yicheng People's Hospital

Ling Gan ( $\nabla$ gl_echo@163.com )

Hubei University of Medicine

\section{Research Article}

Keywords: IDH1 mutation, miR-200c/141, Epithelial-mesenchymal transition, WGCNA, Immune system

Posted Date: January 21st, 2022

DOI: https://doi.org/10.21203/rs.3.rs-1251637/v1

License: (c) (i) This work is licensed under a Creative Commons Attribution 4.0 International License.

Read Full License 


\section{Abstract}

Gliomas are the most common intracranial tumors with a highly invasive nature and poor prognosis. Different stages of gliomas have distinct alterations in genome, genetic and epigenetic changes. Here, we performed enrichment analysis and weighted gene correlation network analysis (WGCNA) on the Cancer Genome Atlas (TCGA) RNA-seq dataset to identify core genes and potential pathways involved in Isocitrate Dehydrogenase 1 (IDH1) mutant lower-grade gliomas (LGG). The IDH1-mutant LGG patients were found to frequently co-occur with abundant genetic alterations, surprisingly, the miR-200c/141 remain unchanged. Although located in close proximity, the expression level of miR-200c was more affected by methylation level and copy number variation than that of miR-141 in IDH1-mutant LGG. GO analysis indicated the high correlation between miR-200c and immune response. Gene set enrichment analysis (GSEA) suggested that leukocyte activation, inflammatory response, lymphocyte activation and adaptive immune response were significantly up-regulated in miR-200c high-expression group. Enrichment analysis demonstrated that leukocyte activation, immune response, lymphocyte proliferation, cell-cell adhesion and other items were participants in IDH7-mutant LGG samples. Furthermore, WGCNA yielded 33 significant modules, including the yellow module which contains genes involved in the immune response. Taken together, these results proposed a promising new therapeutic strategy for treating LGG gliomas through a miR-200c-mediated immune response pathway.

\section{Introduction}

Glioma, which originates from the glial cells, is the most common and aggressive primary brain tumor, accounting for the majority of brain-related deaths[1, 2]. Based on the 2007 World Health Organization (WHO) classification of central nervous system (CNS) tumors, gliomas were categorized by cell type and graded from I to IV (lowest to highest invasiveness)[3]. Benign Grade I glioma, as well as grade II glioma with a survival time of more than 5 years, were classified as low-grade gliomas. While grade III glioma with a survival time of 2-3 years and IV gliomas (Glioblastoma, GBM) were defined as high-grade gliomas[4]. Traditionally, grade II gliomas were considered to have a better prognosis than grade III gliomas[5]. However, with the identification of key molecular alterations such as isocitrate dehydrogenase (IDH) $1 / 2$ mutations in more than half of the grade II and III gliomas, WHO introduced a new classification of the CNS tumors in 2016, and the term "lower-grade gliomas (LGG)" that covers grade II and III gliomas gradually replaced the term "low-grade gliomas"[6]. IDH1 mutations were first discovered in $12 \%$ of GBM patients through an integrated genomic analysis of human glioblastoma multiforme in 2008[7]. Subsequently, IDH1-mutations were identified in over $70 \%$ of grade II/III glioma and secondary GBM cases[8]. Despite considerable efforts in the past decades toward treating gliomas, the survival rate of patients remains dismally low. There are hundreds of molecular alterations in glioma. Although previous studies have shown that IDH1 mutation is an independent favorable prognostic marker in gliomas [9], our knowledge about its initiation and progression is incomplete. The pharmacologic manipulation of key molecular pathways to improve patients' survival rate remains to be further explored. 
MicroRNAs (miRNAs) are small non-coding RNA molecules that have a significant regulatory capacity on the expression of downstream genes[10, 11]. MiR-200 is a family of tumor suppressor miRNAs consisting of five members (miR-200a, miR-200b, miR-200c, miR-429, and miR-141), which are significantly involved in the inhibition of epithelial-to-mesenchymal transition (EMT), repression of cancer stem cells selfrenewal and differentiation, modulation of cell division and apoptosis, and reversal of chemoresistance. The targets of the miR-200 family include the $124 \mathrm{kDa}$ transcription factors zinc finger $E$ box-binding homeobox 1/2 (ZEB1/2), which act as markers of EMT and increase metastasis by repressing E-cadherin and Lgl-2[12]. Among the miR-200 family members, miR-200c is of particular interest for human health and disease, and it has recently emerged as an important regulator of tumorigenicity and cancer metastasis. However, its role in IDH1-mutant LGG is not definitively clear.

In this article, we performed enrichment analysis and weighted gene correlation network analysis (WGCNA) to explore the mechanisms of miR-200c in IDH1-mutant samples and compared its prognostic value in LGG. The results showed a high correlation between miR-200c and immune response including leukocyte activation, inflammatory response, lymphocyte activation, and adaptive immune response in IDH1-mutant LGG samples, which give us a hint to treat LGG using immune checkpoint receptors. Additionally, WGCNA yielded yellow module is highly associated with miR-200c/miR-141 and contains genes involved in the above immune response. Taken together, these results proposed a promising therapeutic strategy for treating LGG through targeting a miR-200c-mediated immune response pathway.

\section{Materials And Methods}

\subsection{Data acquisition and processing}

The RNA-seq datasets, DNA methylation datasets, and clinical datasets from patients with LGG were acquired from the UCSC Xena browser (https://xenabrowser.net/)[13]. The expression of miR-200c/141 in IDH1 mutant LGG and the corresponding normal tissues were obtained from The Cancer Genome Atlas (TCGA) datasets and Genotype-Tissue Expression Portal[14]. Ensembl database (http://asia.ensembl.org/index.html, GRCH38.p13) was applied to integrate ID into official gene symbols. $\mathrm{R}$ Maftools package was used to analysis of somatic variants in IDH1 mutant. Kaplan-Meier method was used for survival analyses, with a P-value generated via log-rank test.

\subsection{Differential Expressed Genes (DEGs) Visualization}

The DEGs analysis of samples with the highest expression of miR-200c $(n=50)$ and the lowest expression $(n=50)$ was identified using the $R$ limma package, with a $Q$ value $<0.05$ and $A b s(F C)>2$ as cutoff criteria. Volcano plots were generated in R software using the VolcanoPlot package. The Heatmap.2 package was used to visualize the expression heatmap of DEGs. Gene Ontology analysis of DEGs was performed using Panther GO (http://geneontology.org/page/go-enrichment-analysis). The ggplot2 package was applied to visualize the results. GSEA analysis of the highest and the lowest expression of miR-200c was performed by using GSEA 3.0 (http://www.broadinstitute.org/gsea/). The heatmap of different GO clusters was generated by Heatmap. 2 package. 


\subsection{WGCNA of RNA-Seq Data from LGG Patients}

WGCNA R package was performed to, rather than only find the DEGs, identify the associations between miR-200c/141 expression and each module by the 'p. weighted' function. A weighted network adjacency was defined by increasing the parameter $\beta$ which is used to optimize the correlations between genes. The power of $\beta=4$ and scale-free $R^{2}=0.95$ were applied as the soft-thresholding parameter. A hierarchical clustering tree was constructed, with different branches of the tree representing different gene modules. The adjacency matrix was transformed into a topological overlap matrix (TOM). Genes were divided into different gene modules based on the TOM-based dissimilarity measure. Then, the module-trait relationship between the clinical data (gender, laterality, neoplasm histologic grade, histological type), the miR-141/200c, and different modules were analyzed. The gene interaction network was constructed by the String database (https://string-db.org/), and the interactive relationship of all the yellow model networks was calculated and visualized by Cytoscape 3.4.0. GO analysis of the genes in the yellow module was performed using the clusterProfiler package and Metascape[15, 16].

\section{Results}

\subsection{Up-regulated ZEB1/ 2 and down-regulated miR- 200c/141 were found in IDH1-mutant tumors}

IDH1 was frequently characterized in LGG. To determine the relative expression of genes in IDH1-mutant tumors, we first compared gene expression of IDH1 with normal brain comparisons catalogued in the TCGA database. In the LGG mutation landscape, 77\% IDH1 mutation with a list of gene mutations were observed mutually, including ATRX (42\%), CIC (20\%), NOTCH1 (11\%), FUBP1 (9\%), PIK3CA (8\%) and IDH2 (4\%). However, neither miR-200c nor miR-141 mutation was detected (Figure 1a). Kaplan-Meier survival analysis results showed that compared with IDH1 wild-type patients, the overall survival (OS) in IDH1mutant patients was increased, indicating that maintaining the functional stability of miR-200c/141 could possibly play an essential role in improving OS (Figure 1b).

The locations of miR-200c/141 determine the target specification. Among the targets, miR-200c/141 were reported to target the ZEB1/2 transcript factor for degradation[17, 18]. To demonstrate whether the expression of ZEB1/2 impacts miR-200c/141 in IDH1-mutant tumors, we used TCGA datasets to analyze the expression pattern of miR-200c/141 and ZEB1/2. Results showed that compared with IDH1 wild-type tumors, the expression of miR-200c/141 were both down-regulated, while the expression of ZEB1/2 was up-regulated in IDH7-mutant tumors, suggesting that ZEB1/2 might be the functional transcriptional suppressor of miR-200c/141 (Figure 1c-f). This inverse relationship between ZEB1/2 and miR-200c/141 has been previously identified from a panel of human cancer cell lines[18]. Taken together, these results suggested that miR-200c and miR-141 play important roles in IDH1-mutant glioma patients' OS via a double-negative feedback loop with ZEB1/2. 


\subsection{MiR-200c is preferentially up-regulated in IDH1-mutant LGG samples}

MiR-200c and miR-141 are both located on chromosome 12p13 within the same microRNA cluster according to chromosome locations[19]. We performed Pearson Correlation Coefficient analysis to identify the association between miR-200c and miR-141, and the results showed a strong positive correlation between the expression level of miR-200c and miR-141 (Figure 2a, b). Previous studies of DNA promoter alterations from TCGA revealed that the miR-200c/141 cluster is epigenetically regulated by DNA methylation[20]. To determine the mechanism of miR-200c/141 in regulating IDH1-mutant LGG, we investigated the relationship between DNA methylation and miRNAs expression. For this purpose, we extracted a $2 \mathrm{~kb}$ sequence from the upstream region of miR-200c and miR-141 to analyze the DNA methylation status of both genes. Six CpG sites were identified using UCSC Genome Browser on the promoter region (Figure 2c). Since one probe data was inaccessible, we plotted a heatmap relating the other 5 CpG sites (cg16642299, cg24702147, cg00366413, cg27534624, and cg22413603). All probes showed low $\beta$ values $(<0.05)$ and there were no differences in $\beta$ values from the five probes between miR200c and miR-141. The CpG site cg16642299 was chosen for further analysis due to its differentiated expression while the other four sites did not show significant differentiated expression (Figure 2d).

The association of methylation status and miR-200c/141 expression level was next checked in IDH1mutant LGG. The results showed that a high methylation level significantly affected the expression of miR-141, but not as strong as that for miR-200c, suggesting miR-200c may have the preferential ability to suppress gliomas progression independently (Figure 2e, f). Furthermore, the association between copy number variation (CNV) and mRNA expression was investigated to verify the driver in LGG tissues; the expression level of miR-200c was affected a lot by high amplification CNV, while the expression level of miR-141 remained relatively unaffected (Figure $2 \mathrm{~g}, \mathrm{~h}$ ). In total, the expression difference of miR-200c/141 can be attributed to DNA methylation or CNV. These results suggest that miR-200c/141 plays an important role in IDH1-mutant tumors, whereas, miR-200c displays a preferential ability in gliomas repression. Consequently, we chose miR-200c for further analysis.

\subsection{RNAseq analysis on miR-200c high- and low-expressed groups}

GO analysis and GSEA were performed on the miR-200c high- and low-expressed groups to explore the gene network that is highly associated with miR-200c expression. Figure3a shows the workflow of the data collection, analysis criteria, and analysis. Samples used for analysis included 50 IDH1-mutant LGG samples with the highest miR-200c expression and 50 IDH1-mutant LGG samples with the lowest miR200 c expression. Firstly, data from patients with miR-200c expression Q value $<0.05$ and $A b s(F C)>1$ was used for GO analysis. Secondly, we performed GSEA based on RNAseq data from both groups.

The volcano plot indicated the fold change and $P$ values of differentially expressed genes (Figure $3 b$ ) which were further clustered into miR-200c high/low-expressed groups, showing the most significant 
genes (Figure 3c). These differentially expressed genes were analyzed by GO analysis. The biological process showed that miR-200c related genes are of the highest association with neutrophil activation involved immune response. The other processes include neutrophil activation, $T$ cell activation, neutrophil degranulation, neutrophil-mediated immunity, regulation of lymphocyte activation, suggesting a strong correlation between miR-200c and immune response (Figure 4a, b)

GSEA indicated that leukocyte activation, inflammatory response, lymphocyte activation, and adaptive immune response were significantly up-regulated in miR-200c high-expressed group, suggesting the role of miR-200c in activating immune response to prevent diseases (Figure 4c).

\subsection{WGCNA on RNAseq in IDH1-mutant LGG samples}

WGCNA is a data mining method that has been widely used to study biological co-expressed gene networks by non-scale weight network construction and module detection[21]. To verify the gene network that was highly associated with miR-200c expression, WGCNA was applied to further analyze IDH1mutant LGG RNAseq data. We first downloaded RNAseq data from the TCGA database, and the samples of $I D H 1$ mutant were clustered using the average linkage method and Pearson's correlation method. The co-expression analysis was carried out to construct the co-expression network. In this study, the power of $\beta=5$ (scale-free $R^{2}=0.8$ ) was selected as the soft-thresholding parameter to ensure a scale-free network.

The dendrogram shown in Figure 5a were 33 modules detected and the genes clustered, indicating the quantified module similarity (Figure $5 \mathrm{a}$, unassigned genes were categorized into the gray module). The weighted network of identified genes from RNAseq was shown in the heatmap, which depicted the topological overlap matrix among all genes (Figure $5 b$ ). These modules were then identified to be related to the expression of miR-200c by the heatmap of module miR-200c expression associations (Figure $5 \mathrm{c}$ ). The yellow module, which had the most significant relationship with miR-200c expression, was selected for further analysis according to our results (Figure $5 \mathrm{c}$ ). The scatterplot showed genetic significance between the expression of miR-200c and module membership in the yellow module (Figure $5 \mathrm{~d}$ ). The results suggested that genes that highly correlated with the yellow module were also strongly associated with miR-200c expression.

\subsection{Enrichment analysis of genes highly associated with miR-200c expression in IDH1-mutant LGG samples}

The network connections in the yellow module, with weight parameters higher than 0.19 from WGCNA, were loaded into Metascape and analyzed. A variety of proteins were included in the network, such as CD180, ITGAL, SYK, ITGB2, CD53, and LAPTM5 (Figure 6a). High inner connectivity of the gene cluster was depicted by the edge sizes. Finally, the genes from the yellow module with a coefficient higher than 0.5 were selected and underwent $\mathrm{GO}$ enrichment analysis (Figure 6b). Leukocyte activation, immune response, lymphocyte proliferation, cell-cell adhesion, and other items were enriched in the analysis, indicating the key role of miR-200c in immune responses in IDH7-mutant LGG tissues (Figure 6b). Through Pearson correlation, miR-200c was found significantly correlated with molecules associated 
with its function including miR141, MLPH, TTC39A, C1 orf64, and GSTM3, and other immune checkpoint molecules including miR141, CTLA4, LAG3, CD160, and CD276 (Figure 6b).

\section{Discussion}

Glioma is a common nervous system cancer that represents difficulties in completely surgical removal[22]. Along with the resistance to radiotherapy and chemotherapy, glioma has become one of the most malignant tumors[23, 24]. LGGs are infiltrative gliomas that most often occur in the adult cerebral hemispheres, a subset of LGGs stays stable for several years, whereas some progress into GBM within a few months, causing a dismal prognosis. Hence, understanding the molecular mechanisms of glioma initiation and progression is important in developing effective therapeutic strategies for LGG patients. In this study, we identified miR-200c, which belongs to the tumor suppressor miR-200 family, is crucial for IDH1-mutant LGG patients through activating an immune response.

MiR-200c belongs to the miR-200 family[25]. In recent years, an increasing number of studies have shown that miR-200c expression inhibits the progression, invasion, EMT and chemoresistance of a variety of tumors, including non-small cell lung cancer, breast cancer stem cells, prostate cancer and ovarian cancer $[26,27][28,29]$. Furthermore, miR-200c/141 were reported to inhibit the expression of transcription factors ZEB1 and ZEB2, which act as markers of EMT, resulting in the downregulation of E-cadherin expression and reduction of cell adhesion and finally promoting the invasion and metastasis of tumor cells[30, 31]. These results indicated that despite the mutually genetic alterations with IDH1 mutation existing in LGG patients, no miR-200c mutation was detected, which eventually resulted in better overall survival. It is consistent with our results, that is, compared with IDH1 wild-type tumors, IDH1-mutant tumors with a relatively higher ZEB1/2 expression showed a lower expression of miR-200c/141, indicating the vital role of miR-200c as a tumor suppressor through regulating the expression of ZEB1/2. The opposite expression pattern of miR-200c/141 and ZEB1/2 demonstrates the negative regulatory feedback loop between miR-200 family members and ZEB TFs, future studies will be needed to test in LGG samples.

IDH1 mutations are closely related to epigenetic variations. Epigenetic mechanisms, especially DNA methylation, play a decisive role during tumorigenesis and EMT and contribute to the regulation of key factors involved in this process. The hypermethylation in regulatory regions of many tumor suppressor genes leads to their transcriptional silencing (e.g. E-cadherin[32]). MiR-200c and miR-141 are both located on chromosome 12p13, however, Niu et al. identified a transcriptional unit containing miR-200c, but not miR-141, within the CpG Island at position -285. The result of an alternative splicing variant exists to splice out miR-141 and conserve the miR-200c transcript was supported by an in-silico inspection, upon which a high-score splice donor site (boundary exon/intron) was detected between the two miRNAs in position $+373[20$ ]. This data might explain the observation previously made by us and others[20, 21], that miR-200c is usually expressed at higher levels and is more affected by methylation level and copy number variation than miR-141, despite their close location. 
Hypermethylation of $\mathrm{CpG}$ islands in promoter regions contributes to the miR-200c expression[20]. According to the epigenetic methylation level, IDH1-mutant LGGs are normally classified as methylationrich. In our case, the methylation level of the miR-200c gene was detected by the six probes on the CpG island before the transcription start site of miR-200c. Moreover, there is a significant association between methylation level and amplification-type alteration, which were both associated with the expression level of miR-200c. To further understand the molecular mechanism behind the effect of miR-200c in IDH7mutant LGG tissues, we performed RNA-seq analysis, downstream GO analysis, and GSEA. The results illustrated that miR-200c might be an important regulator of the immune response, which was consistent with the previous study that miR-200c can control the inflammatory process and the response to an infection[33-35].

It was shown that miR-200c has the ability to modify the efficiency of TLR4 signaling through the MyD88dependent pathway, thus influencing host innate defenses against microbial pathogens[35]. Rogers, T.J., et al. illustrated that miR-200c led to reduced production of the immune-suppressive metabolite kynurenine directly by targeting TD02. Furthermore, in addition to reversing a classical EMT signature, miR-200c represses many genes encoding immune-suppressive factors containing PD-L1/2, HMOX1, and GDF15[34]. It is also recognized that inflammatory-related miR-200c can sustain innate immune responses through LPS-dependent mechanisms[33], strengthening our result that miR-200c plays a vital role in immune responses, providing a therapeutic strategy via a miR-200c-modulated immune response.

These results were further confirmed by WGCNA and downstream enrichment analysis, showing that the genes in the yellow module played essential roles in immune responses. Besides, the genes highly correlated with miR-200c expression showed intensive connection by the visualization of metascape. A variety of the hub-genes were immune proteins, e.g., CD180, ITGAL, SYK, ITGB2, CD53, and LAPTM5, indicating the high association between miR-200c and immune systems.

\section{Conclusions}

In conclusion, all the data presented above suggested that miR-200c played an essential role in the physiological process of IDHI-mutant LGG. Based on the molecular and clinical analysis, our study elucidated that miR-200c linked tightly to immune responses in IDH1-mutant LGG tissues, and was involved in the survival of $I D H I$-mutant LGG patients through targeting intermediates in signaling pathways essential for innate immune responses. Modulating miR-200c-mediated immune responses may provide a new therapeutic strategy for IDH1-mutant LGG patients.

\section{Abbreviations}

CNS, central nervous system

CNV, copy number variation

DEGs, different expression genes 
EMT, epithelial-to-mesenchymal transition

FC, fold change

GBM, glioblastoma

GO, gene ontology

GSEA, gene set enrichment analysis

IDH1, isocitrate Dehydrogenase 1

LGG, Lower-grade gliomas

OS, overall survival

TCGA, The Cancer Genome Atlas

TOM, topological overlap matrix

WGCNA, weighted gene correlation network analysis

WHO, World Health Organization

ZEB1/2, zinc finger E box-binding homeobox 1/2

\section{Declarations}

Ethics approval and consent of participate

Not applicable.

Consent to publish

Not applicable.

Availability of data and materials

The datasets generated and analyzed during the current study are available in the TCGA Research Network: https://www.cancer.gov/tcga. All data generated or analyzed during this study are included in this published article.

Competing of Interest

The authors declare that they have no competing interests.

Funding 
This work was partially supported by the National Natural Science Foundation of China (Grants number: 81901567), the Natural Science Foundation of Hubei Province (Grants number: 2018CFB112).

\section{Authors' contributions}

Study Initiation, YD, HL, and LG; Conceptualization, HL and LG; Methodology, YD, LL, PL, HL and LG; Investigation, YD, LL and PL; Data Analysis, YD and PL; Writing, YD, LL and PL; Supervision, HL and LG. All authors read and approved the final manuscript. YD and LL contributed equally to this work.

\section{Acknowledgements}

This research was also supported by the Chinese Scholarship Council (PL, 202108080205).

\section{References}

1. Ostrom QT, Bauchet L, Davis FG, Deltour I, Fisher JL, Langer CE, et al. The epidemiology of glioma in adults: a "state of the science" review. Neuro Oncol. 2014;16(7):896-913.

2. Ludwig K, Kornblum HI. Molecular markers in glioma. J Neurooncol. 2017;134(3):505-12.

3. Bai J, Varghese J, Jain R. Adult Glioma WHO Classification Update, Genomics, and Imaging: What the Radiologists Need to Know. Top Magn Reson Imaging. 2020;29(2):71-82.

4. Brandner S, von Deimling A. Diagnostic, prognostic and predictive relevance of molecular markers in gliomas. Neuropathol Appl Neurobiol. 2015;41(6):694-720.

5. Gittleman H, Sloan AE, Barnholtz-Sloan JS. An independently validated survival nomogram for lowergrade glioma. Neuro Oncol. 2020;22(5):665-74.

6. Tom MC, Cahill DP, Buckner JC, Dietrich J, Parsons MW, Yu JS. Management for Different Glioma Subtypes: Are All Low-Grade Gliomas Created Equal? American Society of Clinical Oncology Educational Book. 2019(39):133-45.

7. Parsons DW, Jones S, Zhang X, Lin JC, Leary RJ, Angenendt P, et al. An integrated genomic analysis of human glioblastoma multiforme. Science. 2008;321(5897):1807-12.

8. Yan H, Parsons DW, Jin G, McLendon R, Rasheed BA, Yuan W, et al. IDH1 and IDH2 mutations in gliomas. N Engl J Med. 2009;360(8):765-73.

9. Sanson M, Marie Y, Paris S, Idbaih A, Laffaire J, Ducray F, et al. Isocitrate dehydrogenase 1 codon 132 mutation is an important prognostic biomarker in gliomas. J Clin Oncol. 2009;27(25):4150-4.

10. Ambros V. microRNAs: tiny regulators with great potential. Cell. 2001;107(7):823-6.

11. Lu TX, Rothenberg ME. MicroRNA. J Allergy Clin Immunol. 2018;141(4):1202-7.

12. Feng $X$, Wang Z, Fillmore $R, X i$ Y. MiR-200, a new star miRNA in human cancer. Cancer Lett. 2014;344(2):166-73.

13. Cline MS, Craft B, Swatloski T, Goldman M, Ma S, Haussler D, et al. Exploring TCGA Pan-Cancer data at the UCSC Cancer Genomics Browser. Scientific reports. 2013;3:2652. 
14. Consortium G. Human genomics. The Genotype-Tissue Expression (GTEx) pilot analysis: multitissue gene regulation in humans. Science (New York, NY). 2015;348(6235):648-60.

15. Tripathi S, Pohl MO, Zhou Y, Rodriguez-Frandsen A, Wang G, Stein DA, et al. Meta- and Orthogonal Integration of Influenza "OMICs" Data Defines a Role for UBR4 in Virus Budding. Cell host \& microbe. 2015;18(6):723-35.

16. Yu G, Wang LG, Han Y, He QY. clusterProfiler: an R package for comparing biological themes among gene clusters. Omics: a journal of integrative biology. 2012;16(5):284-7.

17. Korpal M, Lee ES, Hu G, Kang Y. The miR-200 family inhibits epithelial-mesenchymal transition and cancer cell migration by direct targeting of E-cadherin transcriptional repressors ZEB1 and ZEB2. The Journal of biological chemistry. 2008;283(22):14910-4.

18. Park SM, Gaur AB, Lengyel E, Peter ME. The miR-200 family determines the epithelial phenotype of cancer cells by targeting the E-cadherin repressors ZEB1 and ZEB2. Genes \& development. 2008;22(7):894-907.

19. Lewis BP, Burge CB, Bartel DP. Conserved seed pairing, often flanked by adenosines, indicates that thousands of human genes are microRNA targets. Cell. 2005;120(1):15-20.

20. Neves R, Scheel C, Weinhold S, Honisch E, Iwaniuk KM, Trompeter HI, et al. Role of DNA methylation in miR-200c/141 cluster silencing in invasive breast cancer cells. BMC research notes. 2010;3:219.

21. Langfelder P, Horvath S. WGCNA: an R package for weighted correlation network analysis. BMC bioinformatics. 2008;9:559.

22. Xiong L, Wang F, Qi Xie X. Advanced treatment in high-grade gliomas. J buon. 2019;24(2):424-30.

23. Frosina G. DNA repair and resistance of gliomas to chemotherapy and radiotherapy. Molecular cancer research: MCR. 2009;7(7):989-99.

24. Weil S, Osswald M, Solecki G, Grosch J, Jung E, Lemke D, et al. Tumor microtubes convey resistance to surgical lesions and chemotherapy in gliomas. Neuro Oncol. 2017;19(10):1316-26.

25. Vienberg S, Geiger J, Madsen S, Dalgaard LT. MicroRNAs in metabolism. Acta Physiol (Oxf). 2017;219(2):346-61.

26. Sato H, Shien K, Tomida S, Okayasu K, Suzawa K, Hashida S, et al. Targeting the miR-200c/LIN28B axis in acquired EGFR-TKI resistance non-small cell lung cancer cells harboring EMT features. Sci Rep. 2017;7:40847.

27. Shimono Y, Mukohyama J, Nakamura S, Minami H. MicroRNA Regulation of Human Breast Cancer Stem Cells. J Clin Med. 2015;5(1).

28. Oh-Hohenhorst SJ, Lange T. Role of Metastasis-Related microRNAs in Prostate Cancer Progression and Treatment. Cancers (Basel). 2021;13(17).

29. Vescarelli E, Gerini G, Megiorni F, Anastasiadou E, Pontecorvi P, Solito L, et al. MiR-200c sensitizes Olaparib-resistant ovarian cancer cells by targeting Neuropilin 1. J Exp Clin Cancer Res. 2020;39(1):3.

30. Chen ML, Liang LS, Wang XK. miR-200c inhibits invasion and migration in human colon cancer cells SW480/620 by targeting ZEB1. Clinical \& experimental metastasis. 2012;29(5):457-69. 
31. Zhou X, Wang Y, Shan B, Han J, Zhu H, Lv Y, et al. The downregulation of miR-200c/141 promotes ZEB1/2 expression and gastric cancer progression. Med Oncol. 2015;32(1):428.

32. Lombaerts M, van Wezel T, Philippo K, Dierssen JW, Zimmerman RM, Oosting J, et al. E-cadherin transcriptional downregulation by promoter methylation but not mutation is related to epithelial-tomesenchymal transition in breast cancer cell lines. British journal of cancer. 2006;94(5):661-71.

33. Parker MI, Palladino MA. MicroRNAs downregulated following immune activation of rat testis. American journal of reproductive immunology (New York, NY: 1989). 2017;77(6).

34. Rogers TJ, Christenson JL, Greene LI, O'Neill KI, Williams MM, Gordon MA, et al. Reversal of TripleNegative Breast Cancer EMT by miR-200c Decreases Tryptophan Catabolism and a Program of Immunosuppression. Molecular cancer research: MCR. 2019;17(1):30-41.

35. Wendlandt EB, Graff JW, Gioannini TL, McCaffrey AP, Wilson ME. The role of microRNAs miR-200b and miR-200c in TLR4 signaling and NF-KB activation. Innate immunity. 2012;18(6):846-55.

\section{Figures}




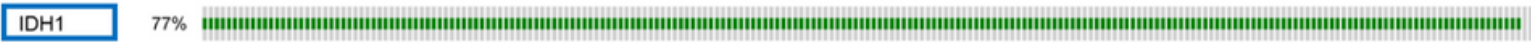

ATRX

$\mathrm{CIC}$

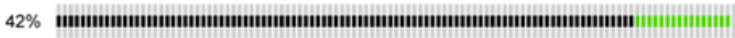

NOTCH1

$20 \%$

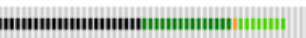

III

$11 \%$ ป

FUbP1 $9 \%$ |

PIK3CA

IDH2

$8 \%$

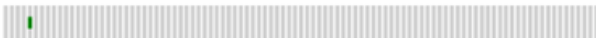

1 ||-|||||||

। 1 |||||||||||||| ||

||||||||||| ||แ!

MIR200C

MIR141

$4 \%$

$0 \%$

Genetic Alteration

" Inframe Mutation (putative driver) || Inframe Mutation (unknown significance) || Missense Mutation (putative driver)

\# Missense Mutation (unknown significance)

- Truncating Mutation (putative driver) No alterations

b
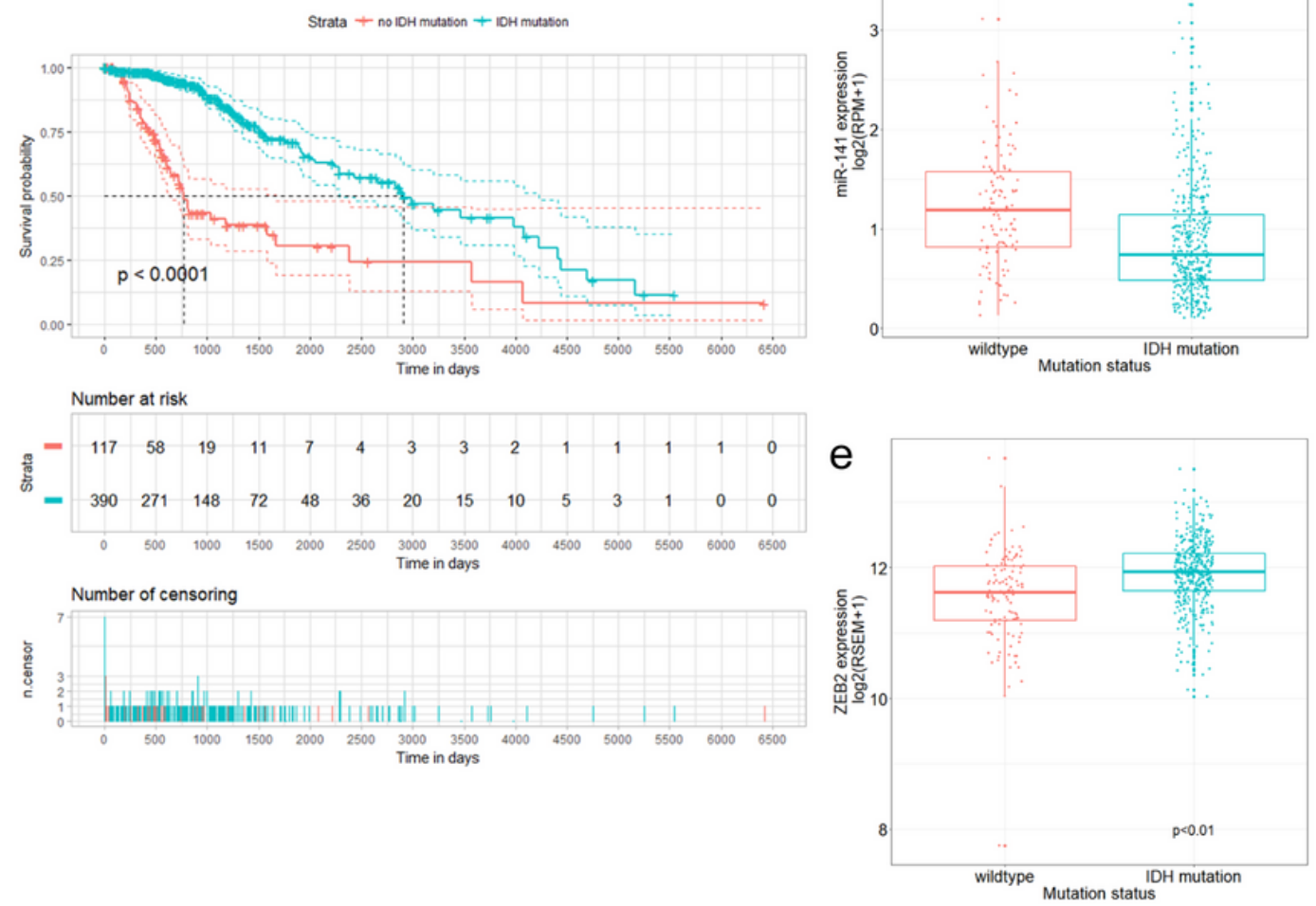
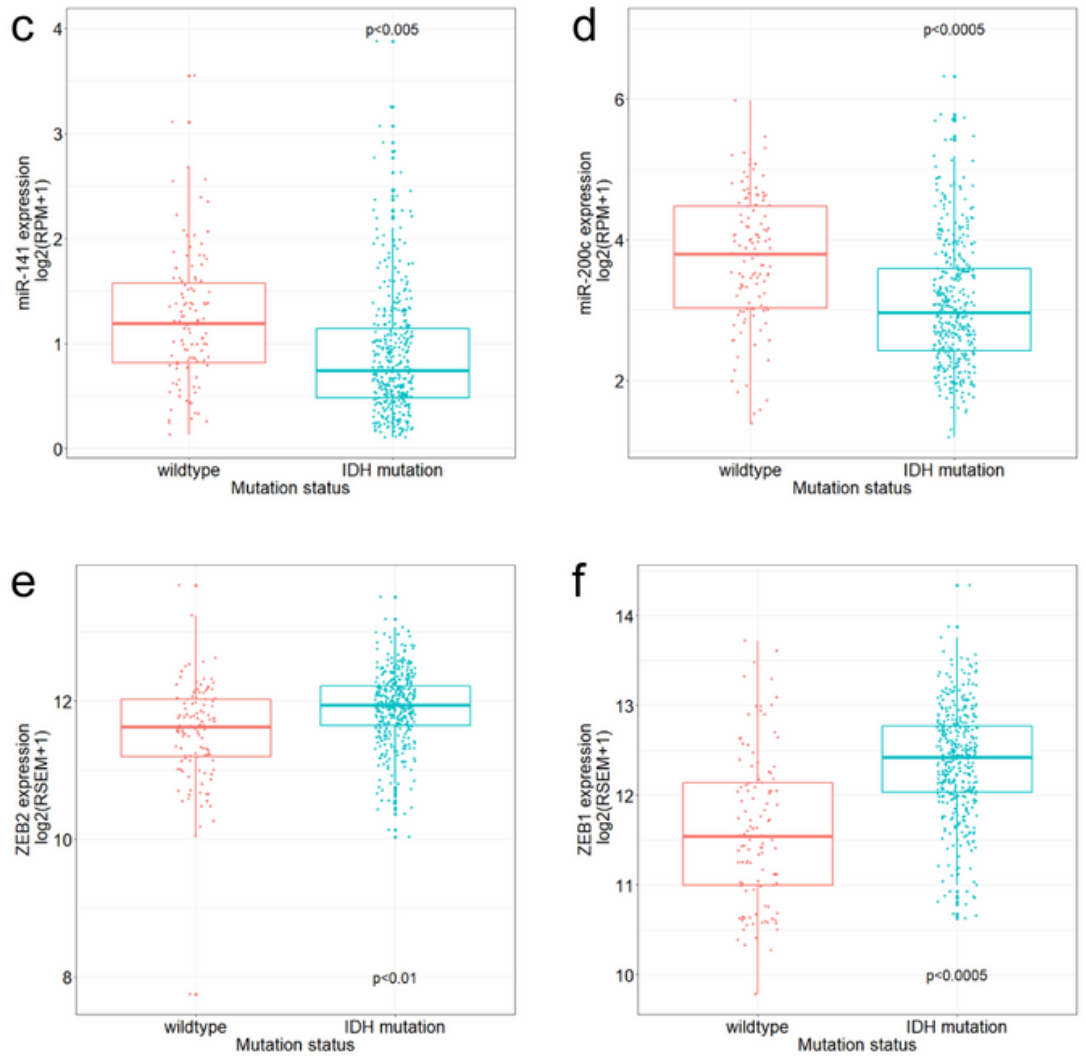

\section{Figure 1}

miR200c/141 mutation, overall survival (OS), miR200c/141 expression andZEB1/2 expression in IDH1mutated samples and corresponding WT. (a) The major mutated genes cluster of IDH1 mutant. Different color lines represent different mutant types. (b) Kaplan-Meier survival analysis of the associations between IDH1-mutant patients and IDH1 wild type patients. (c-f) Boxplots showing the difference of MiR141 (c), MiR-200c (d), ZEB2 (e), ZEB1 (f) expression between IDH1-mutant and WT, respectively. 
a

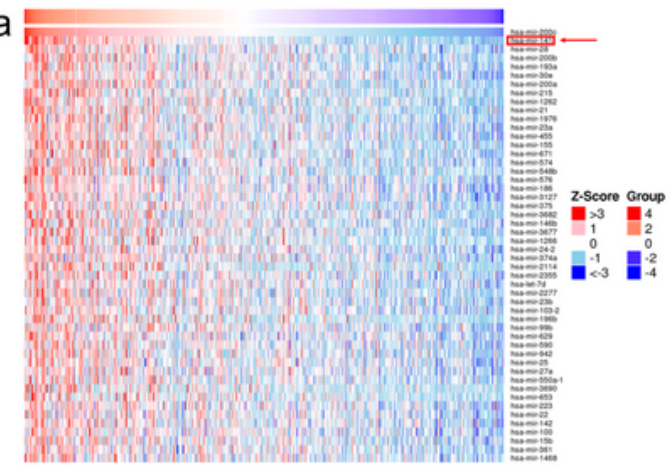

C

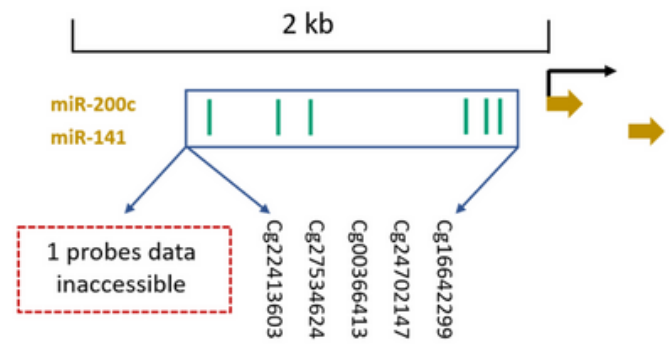

e

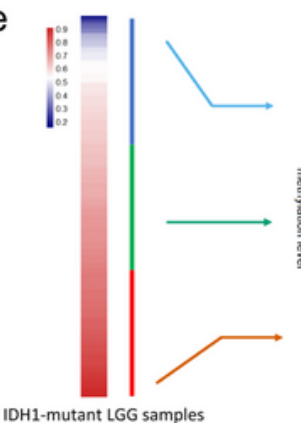

CB 16642299 (B value)

g

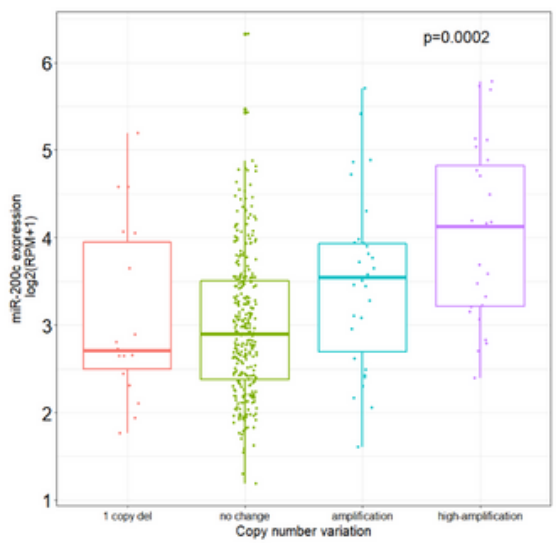

b

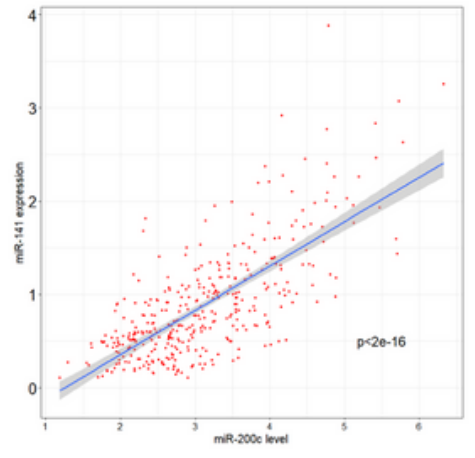

d
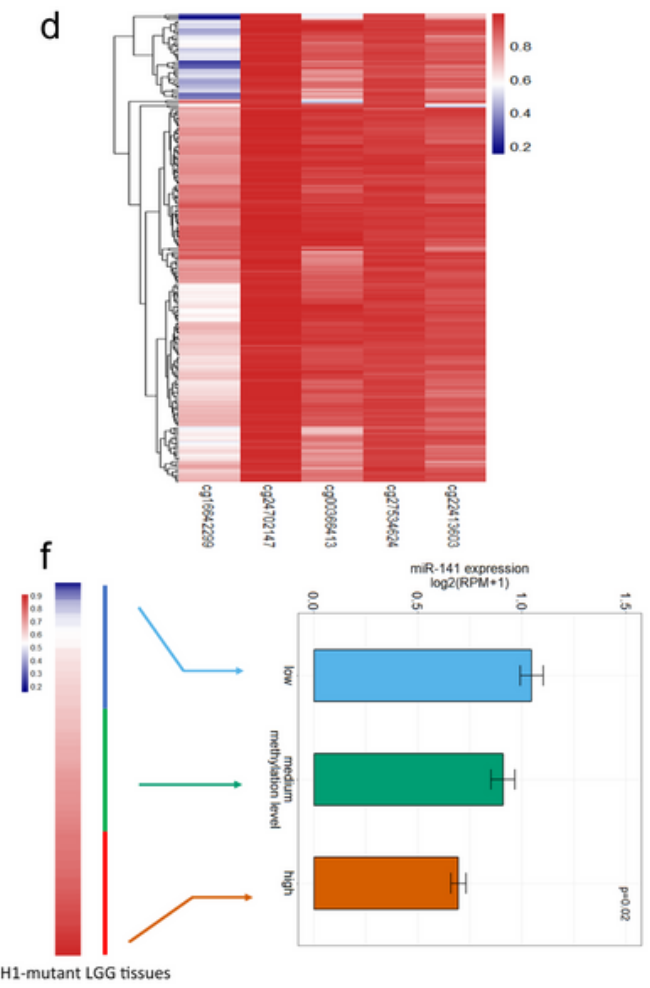

Cg 16642299 ( $\beta$ value)

$\mathrm{h}$

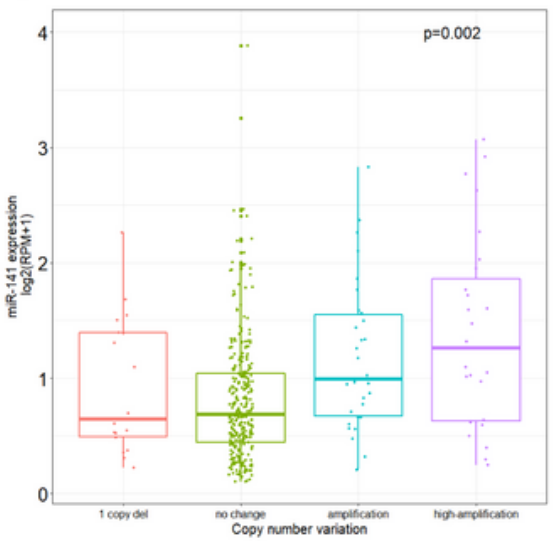

Figure 2

Gene expression association, DNA methylation and CNV analysis of miR-200c/141 in IDH1-mutant LGG samples. (a) The expression correlation between miR-200c and miR-141. The red arrows indicated that miR-141 was the most similar pattern with miR-200c. (b) Scatter plots of Pearson Correlation Coefficient analysis showing the correlation between miR-200c and miR-141. (c) Illustration of five DNA methylation sites of $2 \mathrm{~kb}$ upstream region of miR-200c and miR-141. (d) Differentiated expression analysis of five DNA 
methylation sites. (ef) The relationship between miR-200c/141 expression level and methylation status of cg16642299. (g-h) Boxplots showing the statistical discrepancy between copy number variation (CNV) and miR-200c/141, respectively.

a

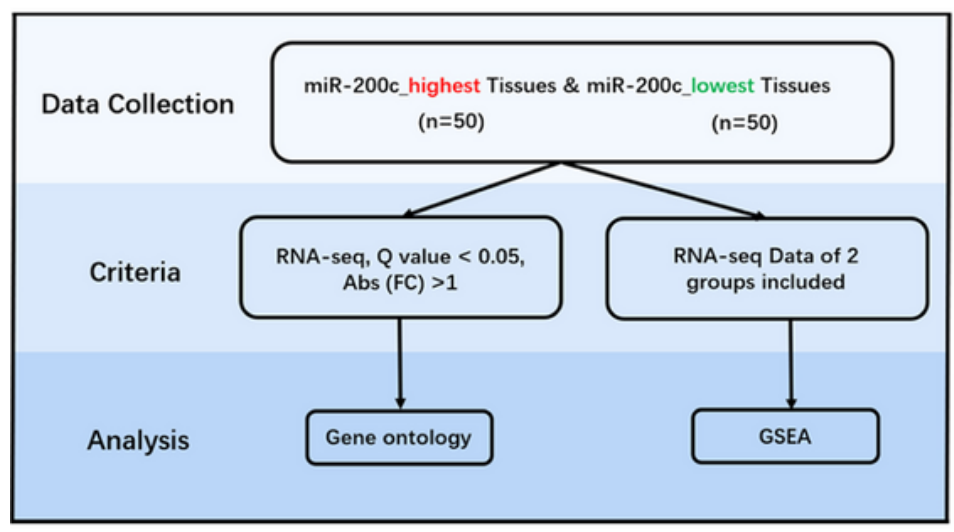

C

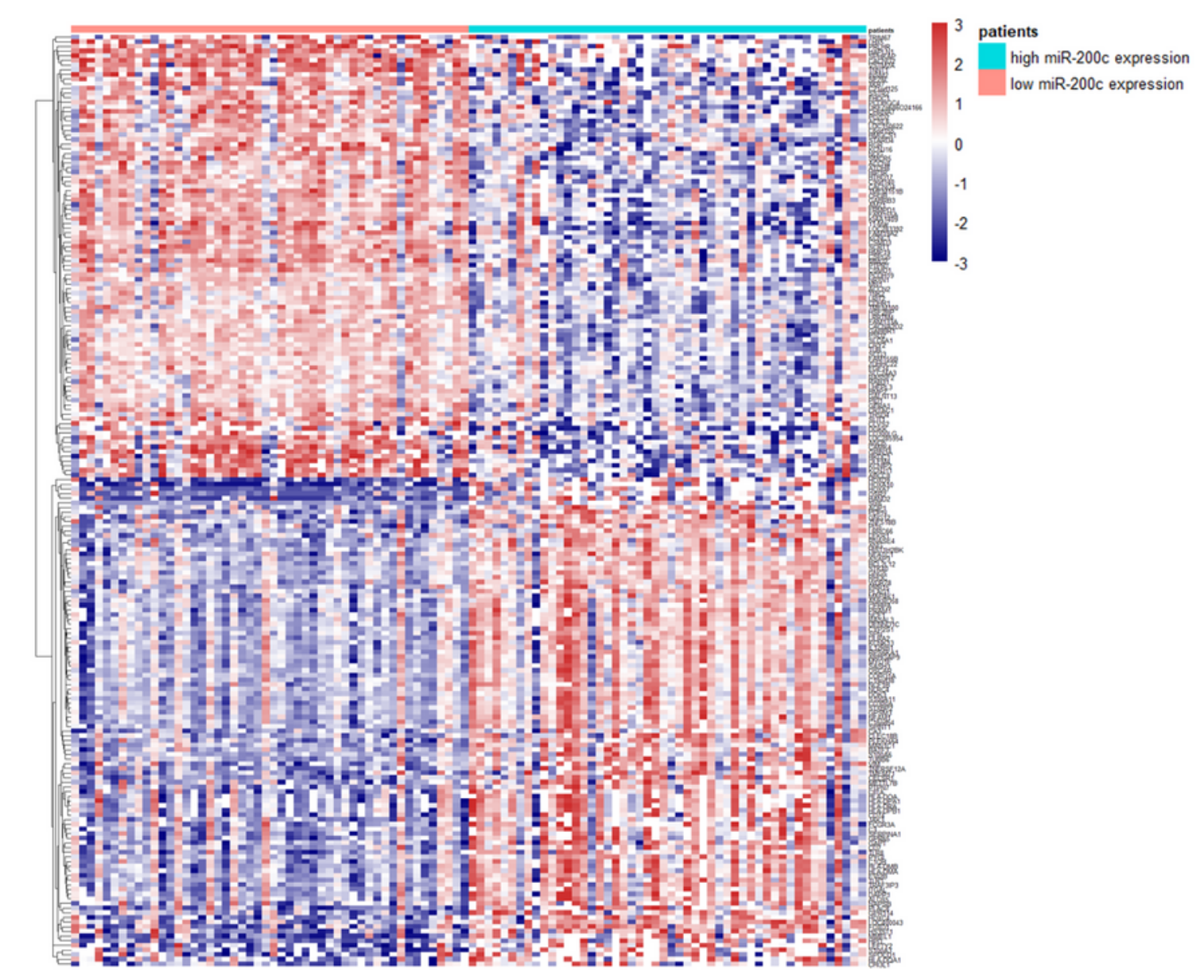

b

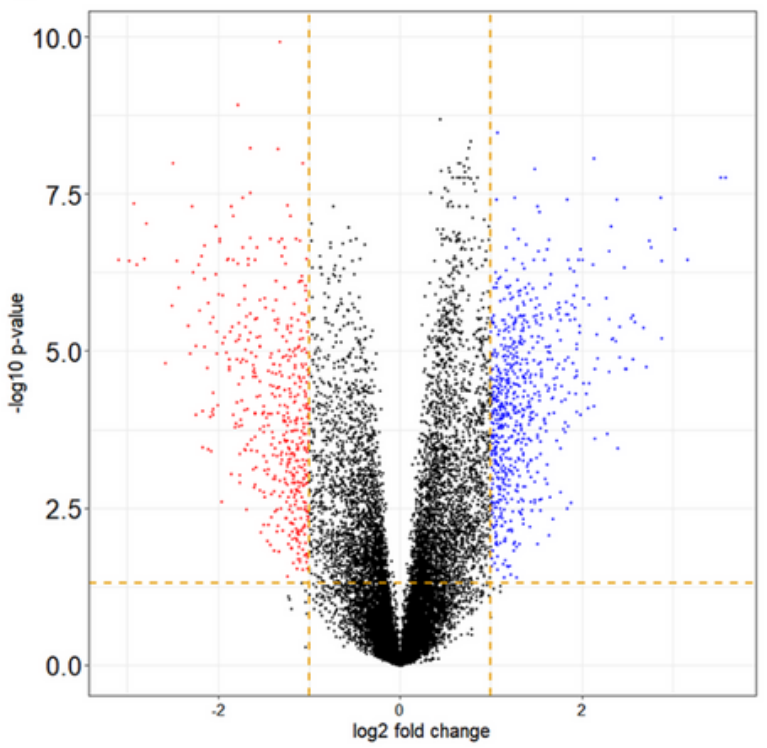

Figure 3 
RNA-seq analysis on miR-200c high- and low- expression IDH1-mutant LGG samples. (a) Illustration of RNA-seq analysis workflow based on differentially expressed genes (DEG) and all genes in RNA-Seq. (b) Volcano plot of DEGs in the groups with high and low expression of miR-200c. Q-value $<0.05, A b s$ (FC) $>1$. (c) Heatmap of the 200 DEGs with the highest significance levels between miR-200c high and low expression groups.
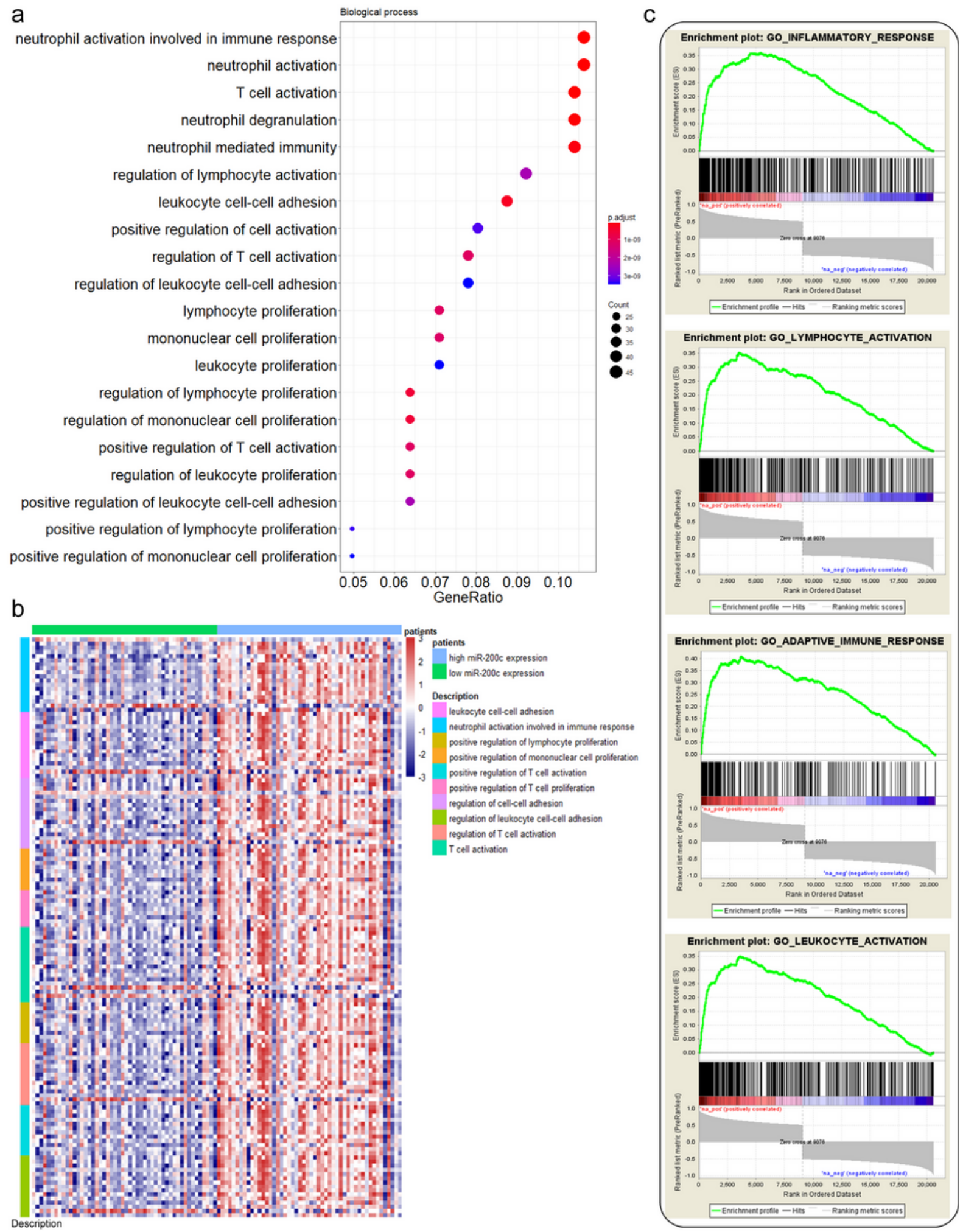

\section{Figure 4}


Gene ontology (GO) and gene set enrichment analysis (GESA) analysis of the DEGs based on the gene expression in RNA-Seq. (a) Functional enrichment annotation of the DEGs. The size of the circle indicates the number of genes. The color of the circle showed the $p$. adjusted value of each annotation. (b) Heatmap of different GO clusters for biological processes. (c) GSEA analysis of the groups with high and low miR-200c expression.

a

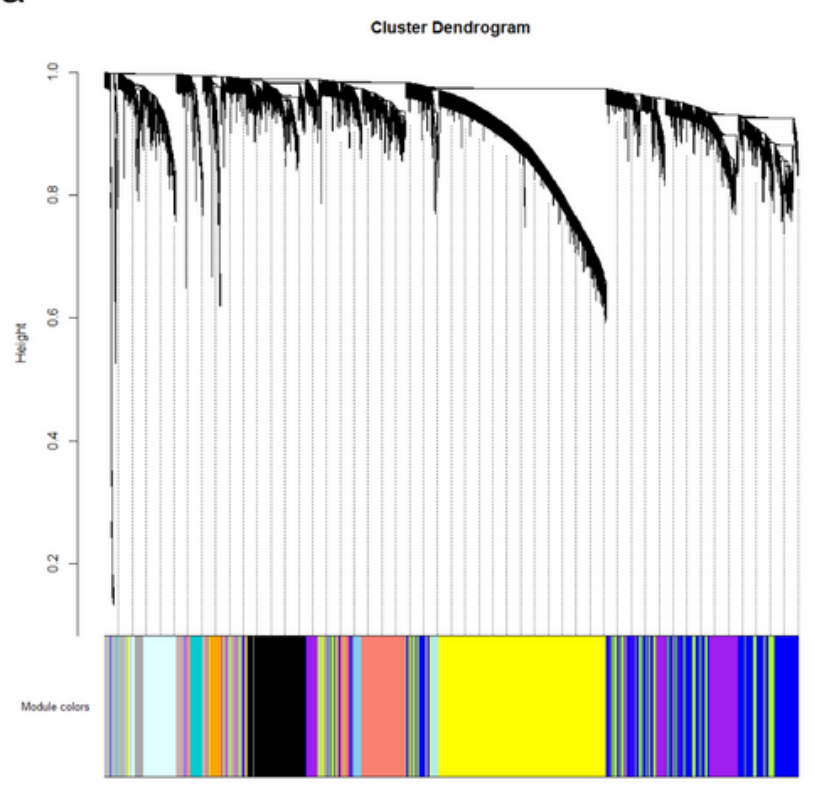

C

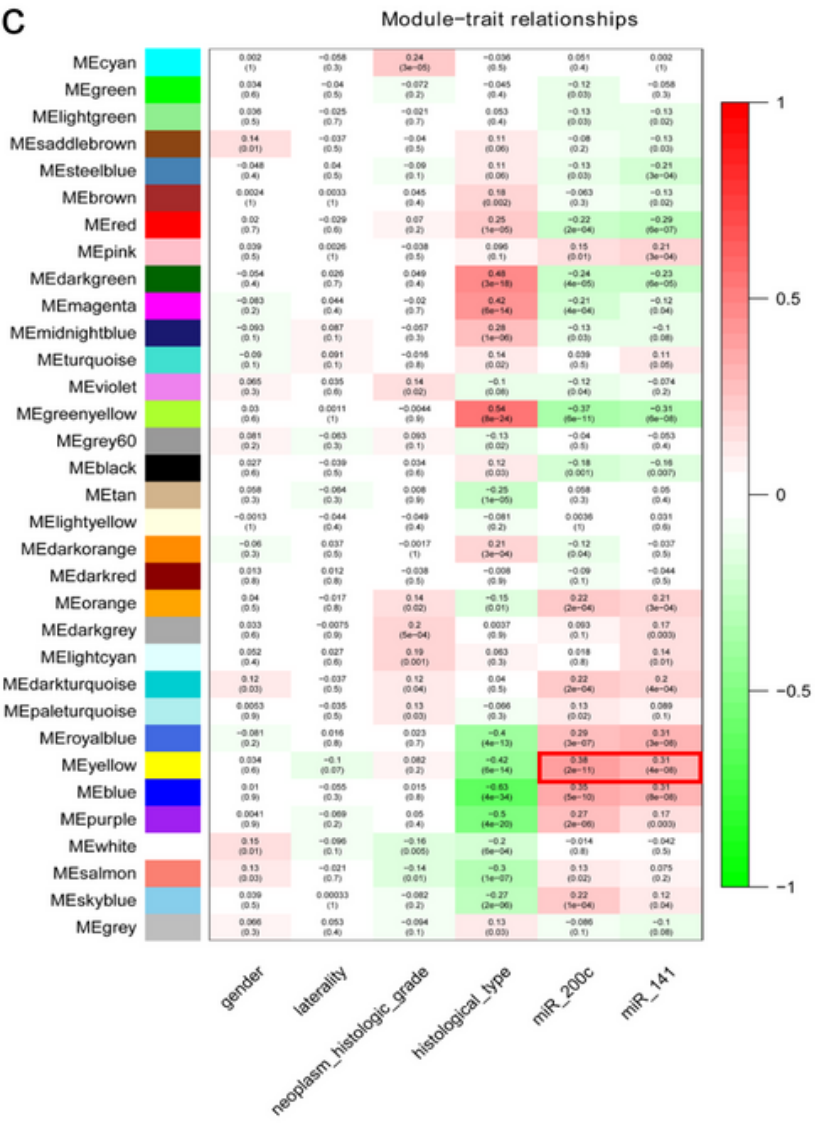

b

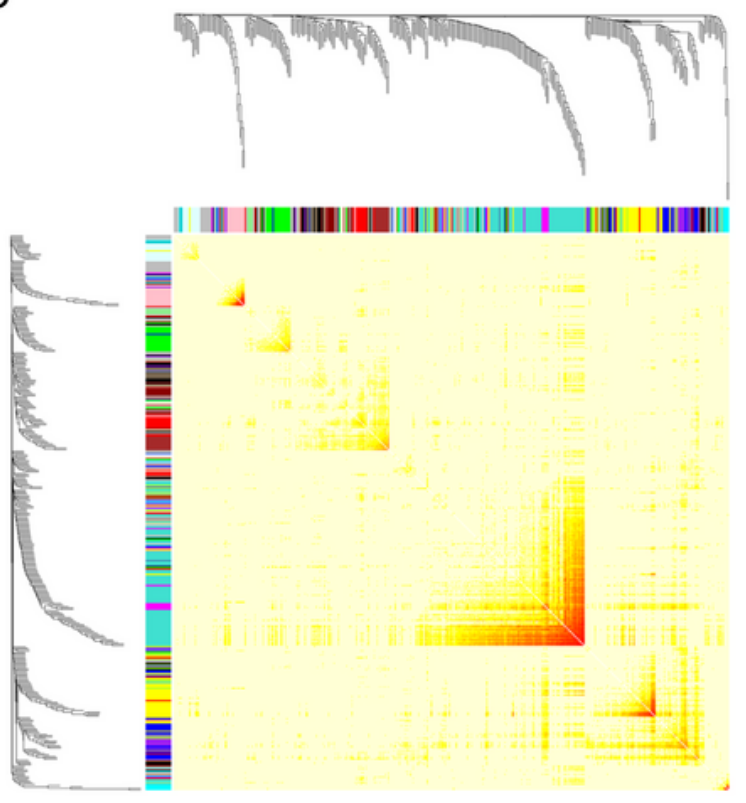

d

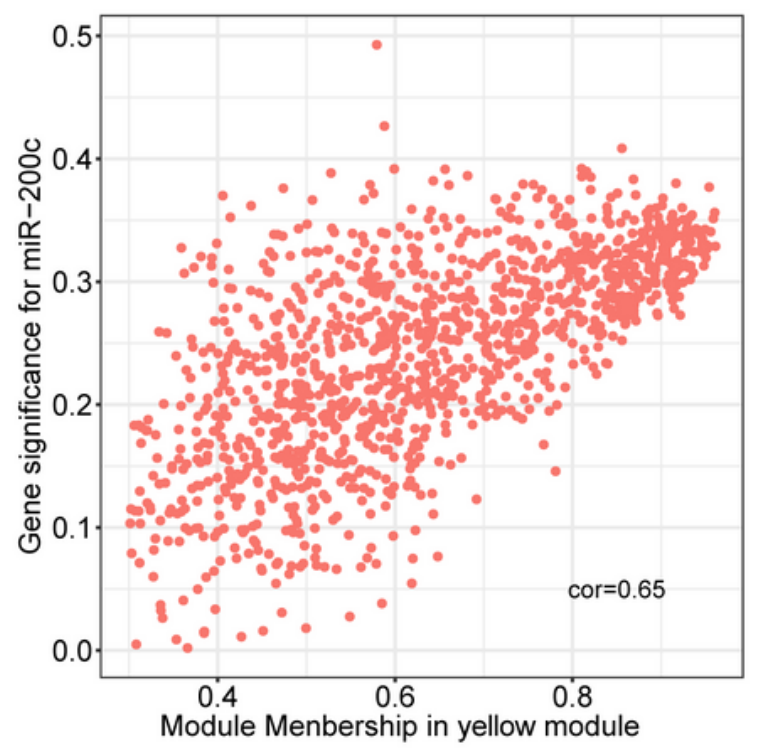

Figure 5 
Identification of the key module of $I D H 1$-mutant by weighted gene co-expression network analysis (WGCNA). (a) Cluster dendrogram of IDH1-mutant, modules identified by WGCNA were assigned as indicated colors in the horizontal bar beneath. (b) Topological overlap matrix of WGCNA detected probes from RNA-Seq. (c) Heatmap of the correlation between different modules. The red box indicated the highest correlation between ME yellow and miR-200c/141. (d) Scatterplot of the genetic significance between miR-200c expression and module membership in the yellow module.

a
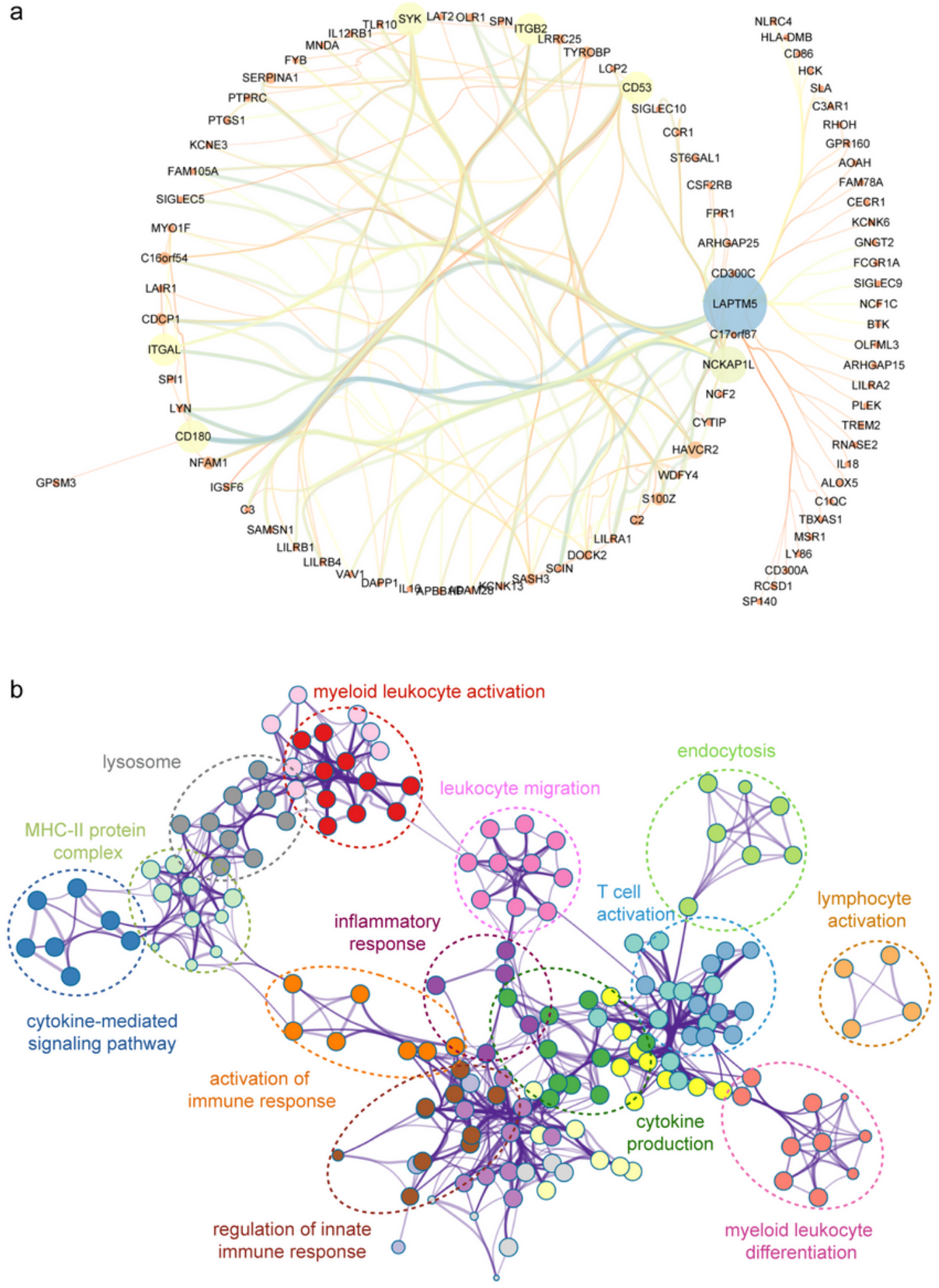


\section{Figure 6}

The network connections between miR-200c and genes in the yellow module. (a) Visualization of the correlation of miR-200c and the most closely related genes in the yellow module. Node sizes reflect the betweenness centrality and edge sizes show the connection weight between genes. (b) GO enrichment analysis network for biological processes, molecular roles and cellular components constructed by all genes in the yellow module. Genes were divided into some subnets according to the connection degree. 